\title{
A New Parameters Identification Technique for Constrained Robot
}

\author{
Kadhim H. Hassan \\ Southern Technical University \\ Basra Engineering Technical \\ College
}

\author{
W. A. Wali \\ University of Basra \\ College of Engineering
}

\author{
T. Y. Abdalla \\ University of Basra \\ College of Engineering
}

\begin{abstract}
This paper presents an identification method for the dynamic parameters of constrained robot manipulator in presence of uncertainties in constraints and robot model. Closed-loop dynamic error equations are derived based on computing input torque and end-effector contact force to survey both desired path and force. The reduced form system equation is adopted for the robot model to prove the proposed identification method. In this work, an ANFIS network has been used and trained based on the presented error component equations to identify the dynamic parameters of two links constrained robot manipulator.
\end{abstract}

\section{Keywords}

Constrained robot manipulator, Robot Model

\section{INTRODUCTION}

Constrained robots are now widely used in various industrial areas for carrying out many practical tasks where the endeffector of the robot has to perform operations on constrained surface ranging from material handling and assembly to welding, cutting and painting $[1,2]$.

Indeed, parameters identification of constrained robot is regarded as an important problem since these parameters are not directly measurable or easily available from the manufacturers due to complex structure and payload of constrained robot during different operation conditions.[3]. A large number of algorithms are adopted to deal with identification of robot model parameters and they resulted, in this field, with different levels of accuracy because most of these algorithms neglect some dynamics of the robot, which is considered as the main drawback of these algorithms [4].

The unknown model parameters have been derived depend on prior knowledge by several approaches such as Least Square Estimation [5]. Dynamic identification is proposed in [6] from the torque data based on weighted least square method. In [7] the maximum likelihood estimation technique for parameters identification is presented. Recently some methods of identification are reported using artificial intelligent such as particle swarm optimization (PSO) [8] and artificial bee colony ( $\mathrm{ABC}$ ) algorithm is used to obtain the unknown robot parameters [9].

In this paper, the dynamic model of constrained robot manipulator is identified based on computed torque method for a two links constrained robot. Two components of dynamic error equations are derived and fed as inputs to the ANFIS model while the outputs are the unknown robot dynamic parameters.

\section{ROBOT MODEL ANALYSIS}

The general equation of the manipulator dynamics with $n$ degree for constrained robot is as below [10]:

$$
M(\theta) \ddot{\theta}+C(\theta, \dot{\theta}) \dot{\theta}+G(\theta)=U+J^{T}(\theta) \lambda
$$

Where $M(\theta) \in R^{n \times n}$ the inertia matrix of the system is, $C(\theta, \dot{\theta}) \in R^{n \times n}$ is the Coriolis and centrifugal terms.

$G(\theta) \in R^{n}$ the gravitation vector, $U \in R^{n}$ denotes the vector of input torque. $\lambda \in R^{m}$ is the contact force.

The constrained force is given by:

$f=J^{T}(\theta) \lambda$

Where $J$ is the Jacobean matrix. The vector $(\theta) \in R^{n}$ denotes the displacement of $\mathrm{n}$ - degree robotic manipulator. The description of constrained surface function on which the manipulator hand lies can be defined as:

$\phi(\theta)=0$

Here $\phi(\theta) \in R^{m}, \mathrm{~m}$ is the number of constraints with $m<n$. Define a vector partition $\boldsymbol{\theta}$ in two vectors, an $(m \times 1)$ vector as $\theta_{1}$ and an $((n-m) \times 1)$ vector $\theta_{2}$, so that the Jacobean matrix can be divided as below:

$$
\begin{aligned}
& J(\theta)=\left[\begin{array}{ll}
J_{1}(\theta) & J_{2}(\theta)
\end{array}\right] \\
& J_{1}(\theta)=\frac{\partial \phi}{\partial \theta_{1}} \quad ; \quad J_{1}(\theta) \in R^{m \times m} \\
& J_{2}(\theta)=\frac{\partial \phi}{\partial \theta_{2}} \quad ; \quad J_{2}(\theta) \in R^{m \times(n-m)}
\end{aligned}
$$

The rank of $J_{l}$ is $m$. Then the constraint equation (2.3) can be represented in variable $\theta_{2}$ only, in terms of a differential function $\psi$

$$
\theta_{1}=\psi\left(\theta_{2}\right) \Rightarrow \phi\left(\psi\left(\theta_{2}\right), \theta_{2}\right)=0
$$

For ease of developing a closed loop control equations, a transformation is introduced so that the constrained robot model can be written in a simpler form. Define a vector $z$ and its partition as: $z_{1} \in R^{m} \quad, z_{2} \in R^{n-m}$

$$
Z=\left[\begin{array}{l}
z_{1} \\
z_{2}
\end{array}\right]=\left[\begin{array}{c}
\theta_{1}-\psi\left(\theta_{2}\right) \\
\theta_{2}
\end{array}\right]=z(\theta)
$$

Which has inverse transformation in term of: $\theta_{(z) \text { : }}$

$\theta=\left[\begin{array}{l}\theta_{1} \\ \theta_{2}\end{array}\right]=\theta(z)=\left[\begin{array}{c}z_{1}+\psi\left(z_{2}\right) \\ z_{2}\end{array}\right]$

The differentiation of the above transformation is defined as: 
$T(z)=\frac{\partial \theta(z)}{\partial z}=\left[\begin{array}{lc}I_{m} & \frac{\partial \psi\left(z_{2}\right)}{\partial z_{2}} \\ 0 & I_{n-m}\end{array}\right]$

Satisfying the constrained equation (2.3) in the new coordinates implies:

$z_{1}=0 ; \quad$ and $\quad \dot{z}_{1}=\ddot{z}_{1}=0$

Define an identity matrix $I_{n}$ with its partition as:

$I_{n}=\left[\begin{array}{ll}E_{1}^{T} & E_{2}^{T}\end{array}\right]$ with

$$
E_{1}=\left[\begin{array}{ll}
I_{m} & 0
\end{array}\right] \text { and } E_{2}=\left[\begin{array}{ll}
0 & I_{n-m}
\end{array}\right]
$$

Then

$Z=\left[\begin{array}{l}0 \\ z_{2}\end{array}\right]=E_{2}^{T} z_{2}$

$\dot{Z}=E_{2}^{T} \dot{z}_{2}$

$\ddot{Z}=E_{2}^{T} \ddot{z}_{2}$

Differentiate transformation relation equation (2.9) yields:

$\dot{\theta}=\left[\begin{array}{ll}I_{m} & \frac{\partial \psi}{\partial z_{2}} \\ 0 & I_{n-m}\end{array}\right] \dot{Z}$

$\dot{\theta}=T\left(z_{2}\right) \dot{Z}$

$\ddot{\theta}=T\left(z_{2}\right) \ddot{Z}+\dot{T}\left(z_{2}\right) \dot{Z}$

Rewrite the robot model equation (2.1) in terms of variable $\mathrm{z}$ and multiply by $T^{T}\left(z_{2}\right)$

$$
M^{*}\left(z_{2}\right) E_{2}^{T} \ddot{z}_{2}+C^{*}\left(z_{2}, \dot{z}_{2}\right) E_{2}^{T} \dot{z}_{2}+G^{*}\left(z_{2}\right)=U^{*}+J^{* T}\left(z_{2}\right) \lambda
$$

Where

$$
\begin{aligned}
& M^{*}=T^{T}\left(z_{2}\right) M\left(\theta\left(z_{2}\right)\right) T\left(z_{2}\right) \\
& C^{*}=T^{T}\left(z_{2}\right)\left[M\left(\theta\left(z_{2}\right)\right) \dot{T}\left(z_{2}\right)+C\left(\theta\left(z_{2}\right), T\left(z_{2}\right) \dot{z}\right) T\left(z_{2}\right)\right] \\
& G^{*}=T^{T}\left(z_{2}\right) G\left(\theta\left(z_{2}\right)\right) \\
& J^{*}=\mathrm{T}^{T}\left(z_{2}\right) J^{T}\left(\theta\left(z_{2}\right)\right) \\
& U^{*}=T^{T}\left(z_{2}\right) U
\end{aligned}
$$

Then equation (2.19) can be decoupled in two subsystems:

$E_{1} M^{*}\left(z_{2}\right) E_{2}^{T} \ddot{z}_{2}+E_{1} C^{*}\left(z_{2}, \dot{z}_{2}\right) E_{2}^{T} \dot{z}_{2}+E_{1} G^{*}\left(z_{2}\right)=E_{1} U^{*}+E_{1} J^{* T}\left(z_{2}\right) \lambda$

$E_{2} M^{*}\left(z_{2}\right) E_{2}^{T} \ddot{z}_{2}+E_{2} C^{*}\left(z_{2}, \dot{z}_{2}\right) E_{2}^{T} \dot{z}_{2}+E_{2} G^{*}\left(z_{2}\right)=E_{2} U^{*}$
We used the fact:

$E_{2} T^{T} J^{T} \lambda=0$

To show that, differentiate the function: $\phi(\theta)=0$

$\dot{\phi}\left(\psi\left(\theta_{2}\right), \theta_{2}\right)=\left(\frac{\partial \phi}{\partial \theta_{1}} \cdot \frac{\partial \psi}{\partial \theta_{2}}+\frac{\partial \phi}{\partial \theta_{2}}\right) \dot{\theta}_{2}=\left(\begin{array}{ll}\frac{\partial \phi}{\partial \theta_{1}} & \frac{\partial \phi}{\partial \theta_{2}}\end{array}\right)\left(\begin{array}{c}\frac{\partial \psi}{\partial \theta_{2}} \\ I\end{array}\right) \dot{\theta}_{2}=J(\theta) T\left(\theta_{2}\right) E_{2}^{T} \dot{\theta}_{2}=0$

The equation (2.25) is $m$ system describes the motion of the constrained robot and equation (2.26) is $(n-m)$ system represents the exerted force through the constrained motion.

\subsection{Computed Torque Control Law}

The equation (2.25) and equation (2.26) will be used to derive the control law in computed torque method, so from equation (2.25), the control input $(U)$ will be :

$E_{1} T^{T} U=E_{1} \hat{C}^{*} E_{2}{ }^{T} \dot{z}_{2}+E_{1} \hat{G}^{*}+E_{1} \hat{M}^{*} E_{2}{ }^{T} u_{o x}-E_{1} T^{T} J^{T} u_{o \lambda}$

Also the control input from equation (26) will be:

$$
E_{2} T^{T} U=E_{2} \hat{C}^{*} E_{2}^{T} \dot{z}_{2}+\hat{G}^{*}+E_{2} \hat{M}^{*} E_{2}{ }^{T} u_{o x}
$$

Where $u_{o x}$ and $u_{o \lambda}$ are an auxiliary control inputs to ensure the tracking for constrained motion and force $\hat{M}, \hat{C}$ and $\hat{G}$ are estimated values of $M, C$ and $G$ because the dynamic parameters of the manipulator actually are not known exactly.

The computed torque control inputs can be defined as:

$$
u_{o x}=\ddot{x}_{d}+K_{D}\left(\dot{x}_{d}-\dot{x}\right)+k_{p}\left(x_{d}-x\right)(2.31)
$$

$u_{o \lambda}=\lambda_{d}+K_{\lambda}\left(\lambda_{d}-\lambda\right)+K_{I} \int_{o}^{t}\left(\lambda_{d}-\lambda\right) d \tau$

Where $x_{d}$ and $x$ are the desired and actual motion trajectory, $\lambda_{d}$ and $\lambda$ are the desired and actual contact force. $K_{D}, K_{P}, K_{\lambda}$ and $K_{I}$ are positive constants.

Solving for $U$ by combining the equations (2.29) and (2.30), gives the control law:

$$
\begin{aligned}
U & =T^{-T} \hat{M}^{*} E_{2}{ }^{T}\left(\ddot{x}_{2 d}+K_{D}\left(\dot{x}_{2 d}-\dot{x}_{2}\right)+K_{P}\left(x_{2 d}-x_{2}\right)\right)+T^{-T}\left(\hat{C}^{*} E_{2}{ }^{T} \dot{z}_{2}+\hat{G}^{*}\right) \\
& -J^{T}\left(\lambda_{d}+K_{\lambda}\left(\lambda_{d}-\lambda\right)+K_{I} \int_{0}^{t}\left(\lambda_{d}-\lambda\right) d \tau\right)
\end{aligned}
$$

The above control law can be rewritten below in a terms of $M$, $C, \mathrm{G}$ instead of $M^{*}, C^{*}, G^{*}$

$$
\begin{aligned}
& U=\hat{M} T E_{2}^{T}\left(\ddot{x}_{2 d}+K_{D}\left(\dot{x}_{2 d}-\dot{x}_{2}\right)+K_{P}\left(x_{2 d}-x_{2}\right)\right)+\hat{C} T E_{2}^{T} \dot{z}_{2}+\hat{G}+\hat{M} \dot{T} E_{2}^{T} \dot{z}_{2} \\
& -J^{T}\left(\lambda_{d}+K_{\lambda}\left(\lambda_{d}-\lambda\right)+K_{I} \int_{0}^{t}\left(\lambda_{d}-\lambda\right) d \tau\right)
\end{aligned}
$$




\subsection{Proposed Error Equations}

Define a closed-loop error system in terms of the following:

$e_{0}=x_{1}=\theta_{1}-\psi\left(\theta_{2}\right)=0$

$e_{1}=x_{2}-x_{2 d}=\theta_{2}-\theta_{2 d}$

$e_{2}=\lambda-\lambda_{d}$

Substitute the control law (2.34) into equations (2.25) and (2.26) yields:

$\ddot{e}_{1}+K_{D} \dot{e}_{1}+K_{P} e_{1}=\left(E_{2} T^{T} \hat{M} T E_{2}{ }^{T}\right)^{-1}\left[E_{2} T^{T}\left(\Delta M T E_{2}{ }^{T} \ddot{z}_{2}+\Delta M \dot{T} E_{2}{ }^{T} \dot{z}_{2}+\Delta C T E_{2}{ }^{T} \dot{z}_{2}+\Delta G\right)\right]$

$E_{1} T^{T} J^{T}\left(e_{2}+K_{\lambda} e_{2}+K_{I} \int_{0}^{t} e_{2} d \tau\right)=\left(\Delta M T E_{2}^{T} \ddot{z}_{2}+\Delta M T \dot{E}_{2}^{T} \dot{z}_{2}+\Delta C T E_{2}^{T} \dot{z}_{2}+\Delta G\right)$ $\left[E_{1} T^{T}-E_{1} T^{T} \hat{M} T E_{2}^{T}\left(E_{2} T^{T} \hat{M} T E_{2}^{T}\right)^{-1} E_{2} T^{T}\right]$

Where

$\Delta M=M-\hat{M}, \quad \Delta C=C-\hat{C} \quad$ and $\quad \Delta G=G-\hat{G}$ (2.40)

It is clear when $M=\hat{M}, C=\hat{C}$ and $G=\hat{G}$, the equations (2.38) and (2.39) will be:

$\ddot{e}_{1}+K_{D} \dot{e}_{1}+K_{P} e_{1}=0$

And

$e_{2}+K_{\lambda} e_{2}+K_{I} \int_{0}^{t} e_{2} d \tau=0$

The purpose of this work is to identify the robot parameters when the dynamic error equations $(2,41)$ and $(2.42)$ are zero. In this paper, the motion and force dynamical errors are used by ANFIS network to identify the uncertain dynamic parameters of the constraint robot. The ANFIS capable of analyzing and simulating the mapping relation between input and output data to optimize the given fuzzy inference parameters through learning algorithm.[11]. ANFIS model is designed based on the equations (2.38) and (2.39), to identify the parameters such that the motion error $\left(\ddot{e}_{1}+K_{D} \dot{e}_{1}+K_{\mathrm{P}} e_{1}\right)$ and the contact force error $\left(e_{2}+K_{\lambda} e_{2}+K_{\mathrm{I}} \int e_{2}\right)$ are approached to zero.

\section{TWO LINKS CONSTRAINED ROBOT}

Consider an example of 2-links constrained robot manipulator with a circular path constrained. The components of the robot model are re-arranged as the following:

$$
\begin{aligned}
& M(\theta)=\left[\begin{array}{ll}
b & b+c \cos \left(\theta_{1}\right) \\
b+c \cos \left(\theta_{1}\right) & a+b+2 c \cos \left(\theta_{1}\right)
\end{array}\right] \\
& C(\theta, \dot{\theta})=\left[\begin{array}{ll}
0 & c \dot{\theta}_{2} \sin \left(\theta_{1}\right) \\
-c\left(\dot{\theta}_{1}+\dot{\theta}_{2}\right) \sin \left(\theta_{1}\right) & -c \dot{\theta}_{1} \sin \left(\theta_{1}\right)
\end{array}\right] \\
& G(\theta)=9.81\left[\begin{array}{r}
c \cos \left(\theta_{1}+\theta_{2}\right) \\
a \cos \left(\theta_{1}\right)+c \cos \left(\theta_{1}+\theta_{2}\right)
\end{array}\right]
\end{aligned}
$$

$a, b$ and $c$ are uncertain physical parameters of the robot: $a=\left(m_{1}+m_{2}\right) \ell_{1}^{2}, \quad b=m_{2} \ell_{2}^{2} \quad$ and $\quad c=m_{2} \ell_{1} \ell_{2}$

$\ell_{1}$ and $\ell_{2}$ are the length of the robot links,

$m_{1}$ and $m_{2}$ are the mass of the two links. The constraint is supposed to be a circle which its center coincides with the axis of the first link.

$\phi(p)=P_{1}^{2}+P_{2}^{2}-r^{2}$

So $\Delta M, \Delta C$ and $\Delta G$ can be given as

$\Delta M=\left[\begin{array}{lc}b-\hat{b} & (b-\hat{b})+(c-\hat{c}) \cos \left(\theta_{1}\right) \\ (b-\hat{b})+(c-\hat{c}) \cos \left(\theta_{1}\right) & (a-\hat{a})+(b-\hat{b})+2(c-\hat{c}) \cos \left(\theta_{1}\right)\end{array}\right]$

$$
\begin{aligned}
\Delta C & =\left[\begin{array}{ll}
0 & (c-\hat{c}) \dot{\theta}_{2} \sin \left(\theta_{1}\right) \\
-(c-\hat{c})\left(\dot{\theta}_{1}+\dot{\theta}_{2}\right) \sin \left(\theta_{1}\right) & -(c-\hat{c}) \dot{\theta}_{1} \sin \left(\theta_{1}\right)
\end{array}\right] \\
\Delta G & =9.81\left[\begin{array}{c}
(c-\hat{c}) \cos \left(\theta_{1}+\theta_{2}\right) \\
(a-\hat{a}) \cos \left(\theta_{1}\right)+(c-\hat{c}) \cos \left(\theta_{1}+\theta_{2}\right)
\end{array}\right]
\end{aligned}
$$

Where $\hat{a}, \hat{b}$ and $\hat{c}$ are estimated values of $a, b$ and $c$, if $a=\hat{a}, b=\hat{b}$ and $c=\hat{c}$ then the motion and force error equations (2.38) and (2.39) would be zero. Three ANFIS models are trained and used, each model has two inputs and one output, where the motion error equation and the force error equation are the inputs while each one of the uncertain parameters $\hat{a}, \hat{b}$ and $\hat{c}$ are the outputs. The training data should be produced by obtaining the equation system error system of the robot by changing the amount of $a, b$ and $c$, if the robot parameters are identified precisely, the dynamical error equations (2.38) and (2.39) would be in the form of equation (2.41) and (2.42) respectively.

\section{SIMULATION EXAMPLE}

The example of 2-links constrained robot is simulated with the proposed computed torque control law. The constraint equation of the studded model is given by equation (3.4). The constraint transformation to the joint variables is:

$$
\begin{aligned}
& P_{1}=\ell_{1} \cos \theta_{2}+\ell_{2} \cos \left(\theta_{1}+\theta_{2}\right) \\
& P_{2}=\ell_{1} \sin \theta_{2}+\ell_{2} \sin \left(\theta_{1}+\theta_{2}\right)
\end{aligned}
$$

The constraint equation to the joint space is given by:

$$
\phi(\theta)=\ell_{1}^{2}+\ell_{2}^{2}+2 \ell_{1} \ell_{2} \cos \left(\theta_{1}\right)-r^{2}=0
$$

Which has a unique solution $\theta_{10}$ as:

$$
\theta_{10}=\psi\left(\theta_{2}\right)=\psi\left(z_{2}\right)=\cos ^{-1}\left(\frac{r^{2}-\left(\ell_{1}^{2}+\ell_{2}^{2}\right)}{2 \ell_{1} \ell_{2}}\right)
$$

The Jacobean of $\phi(\theta)$ then given by:

$J^{T}(\theta)=\left[\begin{array}{c}-2 \ell_{1} \ell_{2} \\ \sin \left(\theta_{10}\right) \\ 0\end{array}\right]$

The transformation matrix $T\left(z_{2}\right)$ is:

$T\left(z_{2}\right)=\left[\begin{array}{ll}1 & 0 \\ 0 & 1\end{array}\right]$ 
Apply the transformation of coordinates:

$$
\begin{aligned}
M^{*} & =\left[\begin{array}{lc}
b & b+c \cos \left(\theta_{10}\right) \\
b+c \cos \left(\theta_{10}\right) & a+b+2 c \cos \left(\theta_{10}\right)
\end{array}\right] \\
C^{*} & =\left[\begin{array}{lc}
0 & c \dot{z}_{2} \sin \left(\theta_{10}\right) \\
-c \dot{z}_{2} \sin \left(\theta_{10}\right) & 0
\end{array}\right] \\
G^{*} & =9.81\left[\begin{array}{c}
c \cos \left(\theta_{10}+z_{2}\right) \\
a \cos \left(\theta_{10}\right)+c \cos \left(\theta_{10}+z_{2}\right)
\end{array}\right]
\end{aligned}
$$

The parameters values are set to be:

$\ell_{1}=1 m, \ell_{2}=0.8 \mathrm{~m}, m_{1}=3.5 \mathrm{Kg}, m_{2}=1.5 \mathrm{Kg}, \quad r=0.6$, the controller gains are selected to be $K_{P}=10, K_{D}=10, K_{I}=2$ and $K_{\lambda}=0.2$

considered the desired trajectory as follow:

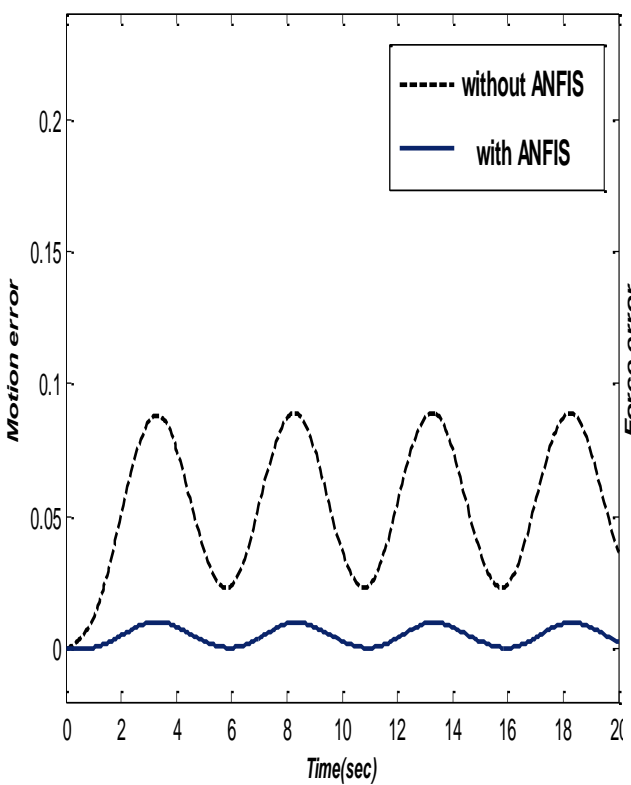

Figure (1) Motion tracking error

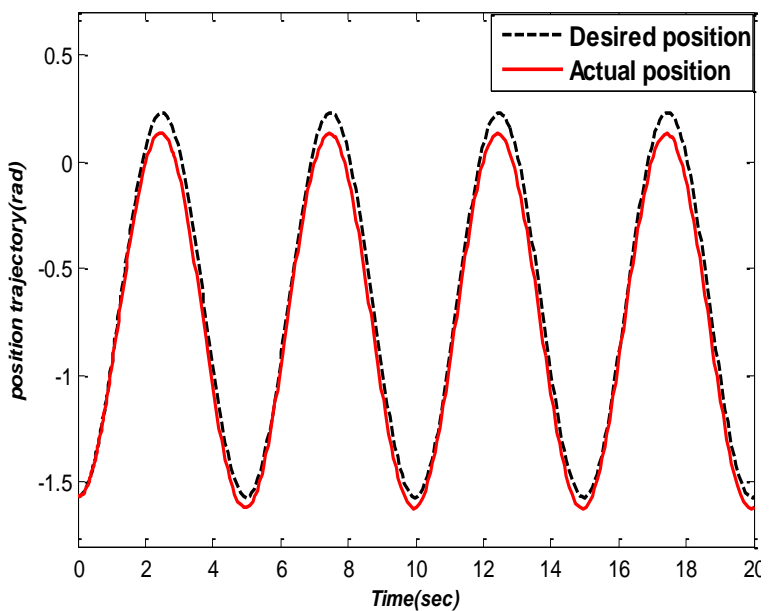

Figure (3) Motion trajectory before identification $\theta_{2 d}=-\frac{\pi}{2}+0.9[1-\cos (1.26 t)]$

the desired contact force is $20 \mathrm{~N}$. Consider the nominal values of the parameters are given as : $a_{o}=5, b_{o}=0.96$ and $c_{o}=1.2$. To include a change in robot parameters, a payload is attached to the second link; the load uncertainty affects the robot dynamic parameters. In order to estimate the model uncertainty, the performance is tested by applying the closed loop control law and sees the tracking error system. Let a mechanical tool of $0.5 \mathrm{Kg}$ handled by the robot hand. The robot is required to track the varying trajectory along the constraint and in the same time exerted a desired force on the surface. The response of the motion tracking error is shown in Figure (1), while the force tracking error is shown in Figure (2). The desired and actual trajectory of constrained robot is illustrated in Figures (3) and (4). It is shown that the proposed technique is able to identify the values of robot parameters; these amounts will make motion and force errors decreasing rapidly

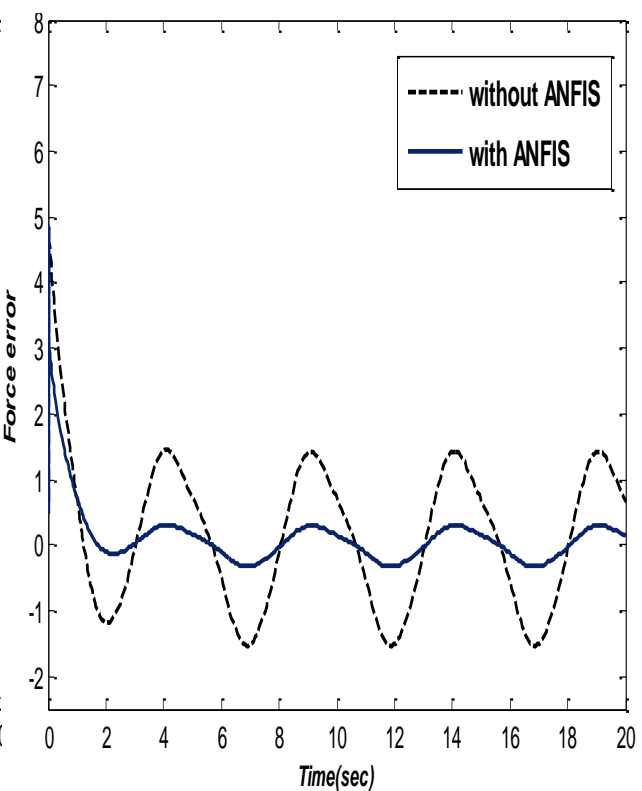

Figure (2) Force tracking error

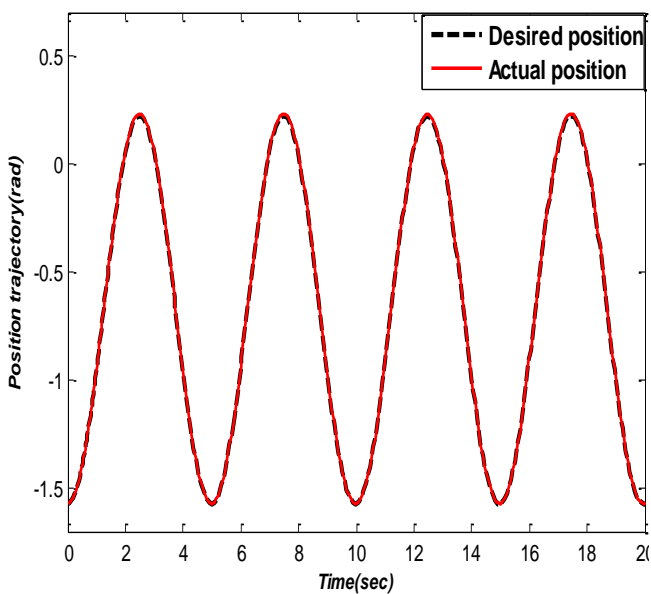

Figure (4) Motion trajectory after identification 


\section{REFERENCES}

[1] J.Wu, J.Wang, Z.You "An Overview of Dynamic Parameter Identification of Robots" Robotics and Computer-Integrated Manufacturing ,Vol.26(5) pp 414419,2010

[2] Y. Chen and F. Dong, "Robot machining: recent development and future research issues," International Journal of Advanced Manufacturing Technology, vol. 66, no. 9-12, pp. 1489-1497, 2013.

[3] J.Swevers, W.Verdonek and J.D.Schutter," Dynamic Model Identification for Industrial Robots" IEEE Control Systems Magazine, Vol.27, no.5, pp 58-71 Sep.2007.

[4] A. Janot, P.O.Vandanjon, and M.Gautier, " A Generic Instrumental Variable Approach for IndustrialRobot Identification" IEEE Trans. On Cirl. Sys. Tech. vol. 22 no. 1, pp. 132-135, Jan 2014.

[5] C. G. Atkeson, C.H. An, and J. M. Hollerbach," Estimation of Inertial Parameters of Manipulator Loads and Links, "International Journal of Robotics and Research, vol. 5, no. 3. Pp.101-119, 1986.

[6] M.Gautier and P. Poignet,, " Extended Kalman Filtering and Weighted Least Square Dynamic Identification of
Robot" Control Engineering Practice, vol.9, no.12, pp1361-1372, 2001.

[7] M. M.Olsen and H.G.Peterson, " A New Method for Estimating Parameters of a Dynamic Robot Model “ IEEE Trans. On Robotics and Automation. Vol. 17, no.1, pp. 95-100, 2001.

[8] Z. Bingul and O.Karahan, " Dynamic Identification of Staubli RX-60 Robot using PSO and LS methods" Expert Systems with Applications. Vol. 38, no. 4, pp.4136-4149, 2011.

[9] S.Chiavenini, B.Siciliano and I.Villani, " A survey of Robot Interaction Control Schemes with Experimental Comparison " IEEE/ASME Transactions on Mechatronics vol.4 issue. 3 , pp. .273-285 Sep. 1999.

[10] L.Ding, H.Wu, Y.Yang "Dynamic model identification for 6-DOF Industrial Robot" Journal of Robotics, Vol.2015 pp 1-9, 2015

[11] M A Denai, F. Palis, and A. Zeghbib "ANFIS Based Modeling and Control of Non-linear Systems: A Tutorial" IEEE International Conference on Systems, Man. and Cybemetics, PP.3433-3438, 2004. 